\title{
ARTICLE
}

\section{Comparing gamma-ray fluxes through multidimensional and equivalent one-dimensional shields}

\author{
Jeffrey A. Favorite* \\ Los Alamos National Laboratory, Los Alamos, NM, 87545, USA
}

\begin{abstract}
For computational efficiency or mere simplicity, it is common to approximate a multidimensional radioactive system with an "equivalent" one-dimensional spherical system. Uncollided decay gamma-ray leakages and neutron-induced gamma-ray leakages from simple multidimensional source/shield systems were compared with those from similar spherically symmetric systems. Maintaining the location and thickness of a flat or cylindrical shield, but making it a sphere, leads to large errors when the shield is close to the source. The spherical shell at the same location as the multidimensional one that was most equivalent, in terms of providing the best match to the line leakages, was up to $23 \%$ thinner (decay gamma rays) or $50 \%$ thicker (neutron-induced gamma rays).
\end{abstract}

Keywords: shielding; gamma rays; one-dimensional transport calculations; multidimensional transport calculations; sphere; cylinder; box

\section{Introduction}

For computational efficiency or mere simplicity, it is common to approximate a multidimensional radioactive system with an "equivalent" one-dimensional spherical system. The appropriateness of this approximation for the calculation of uncollided (decay) gamma rays and neutron-induced gamma rays is examined computationally in this paper. Section 2 looks at uncollided decay gamma rays and Sec. 3 looks at neutron-induced gamma-rays.

\section{Decay gamma rays}

Test configurations for uncollided gamma rays used a solid 10-kg highly enriched uranium (HEU) source $\left(94.73 \%{ }^{235} \mathrm{U}, 5.27 \%{ }^{238} \mathrm{U}\right.$ by weight; density 18.74 $\left.\mathrm{g} / \mathrm{cm}^{3}\right)$. The source was either a sphere with a radius of $5.03169 \mathrm{~cm}$ or a cylinder with its height equal to its diameter of $8.79117 \mathrm{~cm}$. The problems used a series of steel (density $7.86 \mathrm{~g} / \mathrm{cm}^{3}$ ) shells of different geometries (flat wall, cylindrical shell, and spherical shell) and sizes (location and thickness).

Only uncollided decay gamma rays (areas under photopeaks) from uranium were considered; scattering was ignored. Uncollided line fluxes were computed with MCNP [1] using a point detector located $100 \mathrm{~cm}$ from the center of the object. When the object had any cylindrical components, the detector was $100 \mathrm{~cm}$ from the axial centerline (so that the detector was "looking

*Corresponding author. Email: fave@lanl.gov through" the curved cylindrical surface), centered over the source. When the shield was a box, the line connecting the source center with the detector point was orthogonal to one side of the box. In all cases, detector fluxes were converted to approximate line leakages by multiplying by the surface area of an assumed $4 \pi$ detector with a radius of $100 \mathrm{~cm}$.

Figure 1 shows one example, the leakage of the $766-\mathrm{keV}$ line as a function of shield location (inner

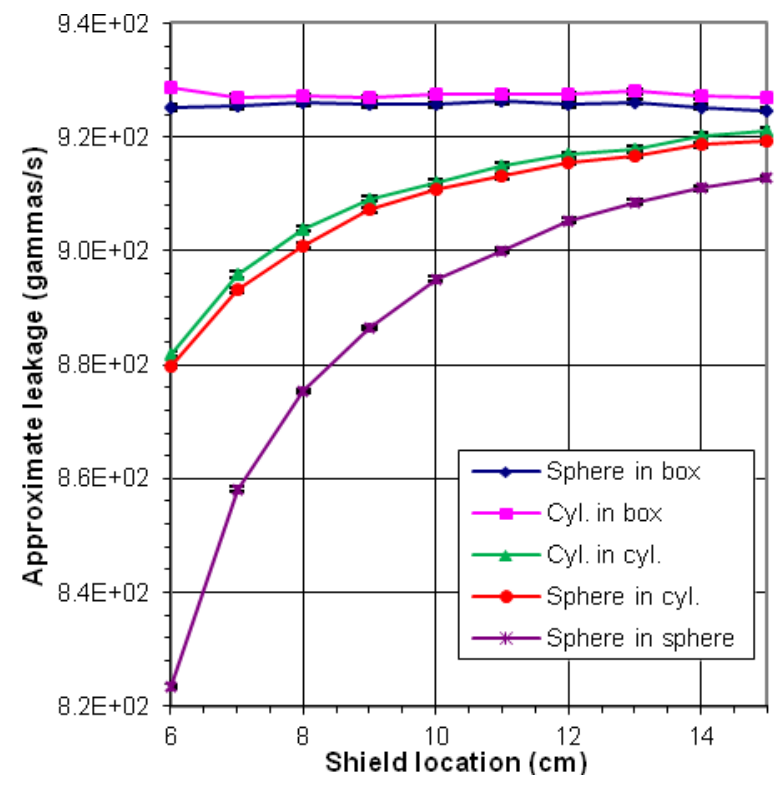

Figure 1. Approximate 766-keV line leakages for various HEU geometries with a shield thickness of $1.0 \mathrm{~cm} .1 \sigma$ Monte Carlo uncertainties are shown. 
dimension) for a 1-cm thick shield. The geometry most sensitive to the location of the shield is the spherical one.

Figure 2 and Figure 3 show the leakage differences between the multidimensional geometries and the one-dimensional spherical geometries, relative to the multidimensional leakages, for the 144- and 1001-keV lines, respectively. Simply approximating a flat or cylindrical shield at location $d$ and of thickness $t$ with a spherical shield of radius $d$ and thickness $t$ results in line fluxes that are underestimated, more for flat, close, or optically thick shields than for cylindrical, far, or optically thin shields; line fluxes were underestimated by as much as $27 \%$ in this study. (The effect of optical

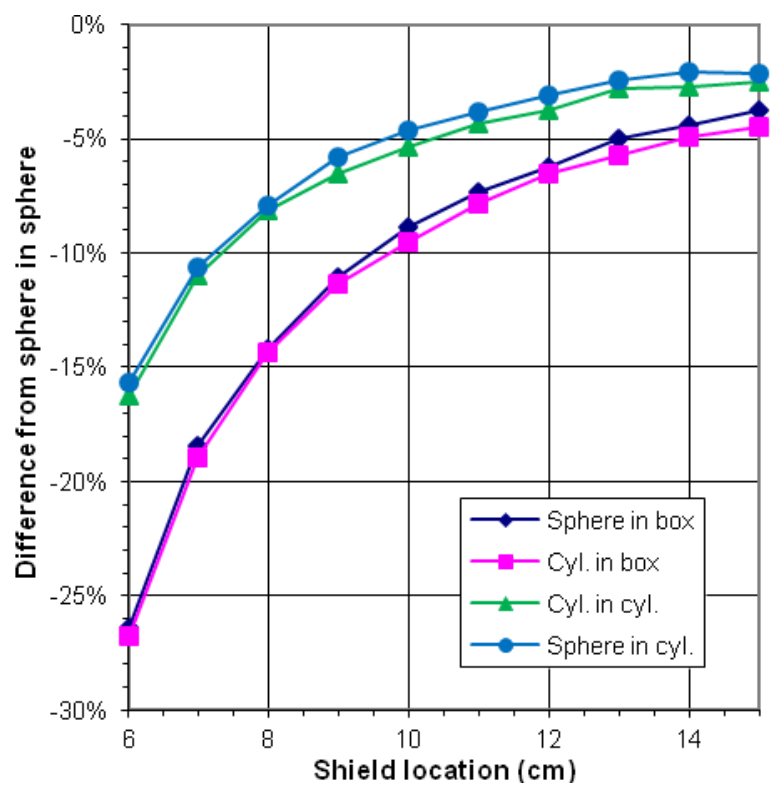

Figure 2. Difference between $144-\mathrm{keV}$ line fluxes from HEU multidimensional geometries and sphere-in-sphere geometries with a shield thickness of $1.0 \mathrm{~cm}$.

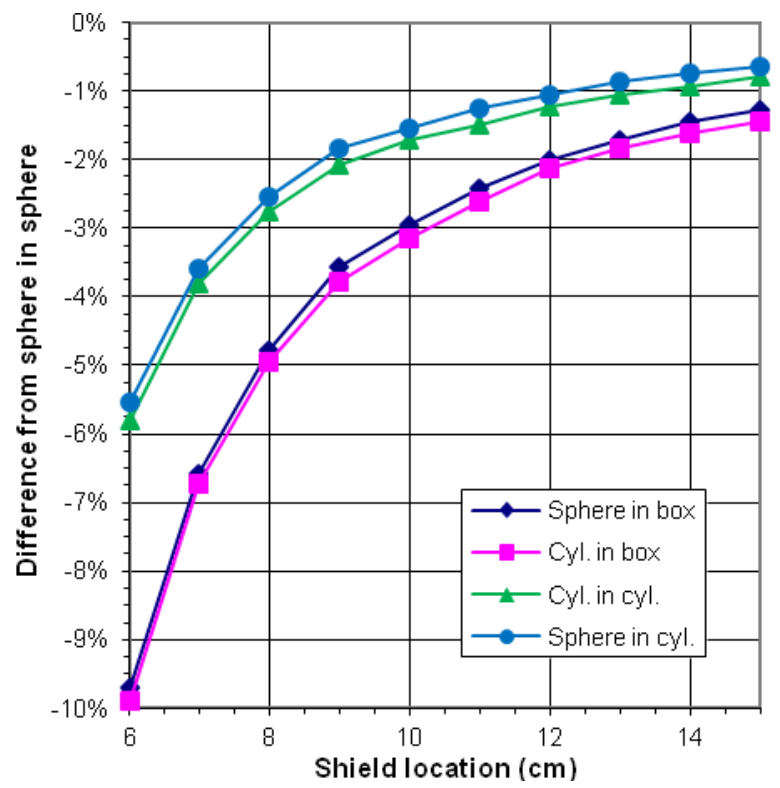

Figure 3. Difference between $1001-\mathrm{keV}$ line fluxes from HEU multidimensional geometries and sphere-in-sphere geometries with a shield thickness of $1.0 \mathrm{~cm}$. thickness, cross section times thickness, is shown in [2].)

What is the "equivalent" one-dimensional spherical representation of a multidimensional object? The optimum spherical representation for each of the multidimensional systems was found by fixing the inner radius of the shield at $d$ and searching for the thickness $t$ that minimized the $\chi^{2}$ difference between the Monte Carlo (reference) leakages and leakages from the spherical object, which were calculated with a deterministic ray-tracing code [3]. The standard $\chi^{2}$ difference is

$$
\chi^{2} \equiv \sum_{g=1}^{G}\left(\frac{R^{g}(t)-R_{0}^{g}}{\sigma_{0}^{g}}\right)^{2}
$$

where $G$ is the number of gamma-ray lines, $R^{g}(t)$ is the calculated one-dimensional response (flux or estimated leakage) for line $g$ given thickness $t$, and $R_{0}^{g}$ and $\sigma_{0}^{g}$ are the reference flux or leakage and its associated standard deviation for line $g . R_{0}^{g}$ and $\sigma_{0}^{g}$ are the MCNP results. The 144-, 186-, 766, and 1001-keV uranium lines were used in the optimization, so $G=4$. $\chi^{2}$ was minimized for each system using the Levenberg-Marquardt method [4].

The difference between the optimal spherical shield thickness (in terms of matching the uncollided fluxes) and the assumed sphere of thickness $t$ (either 0.2 or 1 $\mathrm{cm}$ ) is plotted as a function of shield location $d$ in Figure $\mathbf{4}$ for several geometries. The difference was as high as $23 \%$.

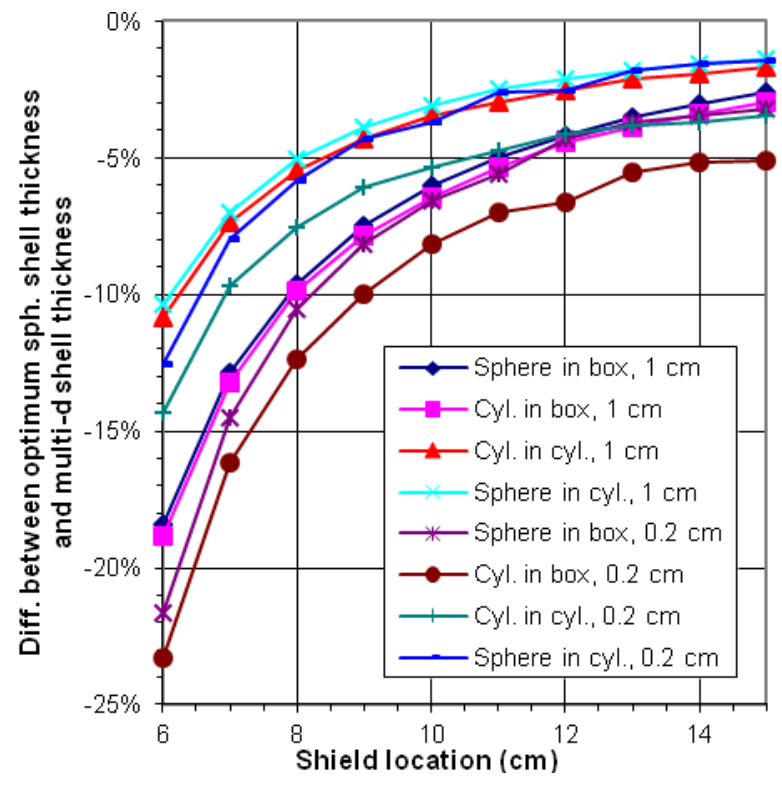

Figure 4. Difference between the optimum spherical shield thickness and the multidimensional shield thickness, relative to the latter, using four decay gamma-ray lines from HEU.

\section{Neutron-induced gamma rays}

Test configurations for neutron-induced gamma-ray line fluxes used a small $(0.001-\mathrm{cm}$ radius $){ }^{252} \mathrm{Cf}$ neutron source (modeled as a void) centered within a series of 
C-H-N-O shells of different geometries (flat, cylindrical, and spherical) and sizes (location and thickness). The C-H-N-O material was $31.12 \%$ natural carbon, $2.79 \%$ ${ }^{1} \mathrm{H}, 29.34 \%{ }^{14} \mathrm{~N}$, and $36.75 \%{ }^{16} \mathrm{O}$ (by weight) and had a density of $1.678 \mathrm{~g} / \mathrm{cm}^{3}$. Calculations were done with MCNP using the continuous-energy version of the ACTI data library [5]. Four neutron-induced gamma rays produced in the $\mathrm{C}-\mathrm{H}-\mathrm{N}-\mathrm{O}$ and their production mechanisms are shown in Table $\mathbf{1 .}$

Table 1. Neutron-induced gamma-ray lines.

\begin{tabular}{cc}
\hline Line energy $(\mathrm{MeV})$ & Source \\
\hline 1.8848 & ${ }^{14} \mathrm{~N}(\mathrm{n}, \gamma)$ \\
2.2233 & ${ }^{1} \mathrm{H}(\mathrm{n}, \gamma)$ \\
3.6840 & ${ }^{12} \mathrm{C}(\mathrm{n}, \gamma)$ \\
4.4390 & ${ }^{12} \mathrm{C}\left(\mathrm{n}, \mathrm{n}^{\prime} \gamma\right)$ \\
\hline
\end{tabular}

Gamma-ray line fluxes were computed with MCNP using a point detector located $100 \mathrm{~cm}$ from the center of the object using the same symmetries as in Sec. 2. Detector fluxes were converted to approximate line leakages as in Sec. 2. These calculations include gamma-ray scattering.

Figure 5 shows one example, the leakage of the 2.2233-MeV thermal capture line as a function of shield location (inner dimension) for a $10-\mathrm{cm}$ thick shield. Leakages from the three geometries were equally sensitive to the location of the shield (their values differ according to the geometry but the shapes of the curves are similar).

Figure 6 and Figure 7 show the leakage differences between the multidimensional geometries and the one-dimensional spherical geometries, relative to the multidimensional leakages, for the $1.8848 \mathrm{-MeV}$ thermal capture line and the $4.4390-\mathrm{MeV}$ inelastic scatter line,

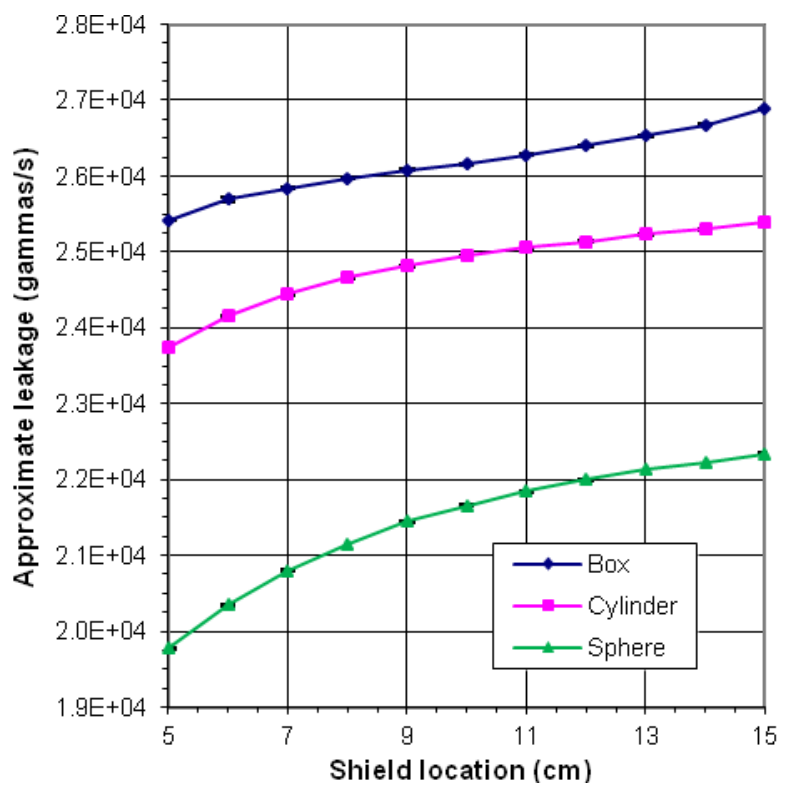

Figure 5. Approximate 2.2233-MeV line leakage for various $\mathrm{C}-\mathrm{H}-\mathrm{N}-\mathrm{O}$ geometries with a thickness of $10 \mathrm{~cm} .1 \sigma$ Monte Carlo uncertainties are shown. respectively. Simply approximating a flat or cylindrical shield at location $d$ and of thickness $t$ with a spherical shield of radius $d$ and thickness $t$ results in line fluxes that are underestimated, more for flat, close, or thin shields than for cylindrical, far, or thick shields; line fluxes were underestimated by as much as $43 \%$ in this study. For this source and shield combination, inelastic scattering lines are much less sensitive than thermal capture lines to the geometry and the shield location (relative to the spherical geometry). The difference in mass between a spherical $\mathrm{C}-\mathrm{H}-\mathrm{N}-\mathrm{O}$ shell and another shape has a huge effect on neutron thermalization.

Again, the optimum spherical representation for each of the multidimensional systems was found by fixing the inner radius of the shield at $d$ and searching for the

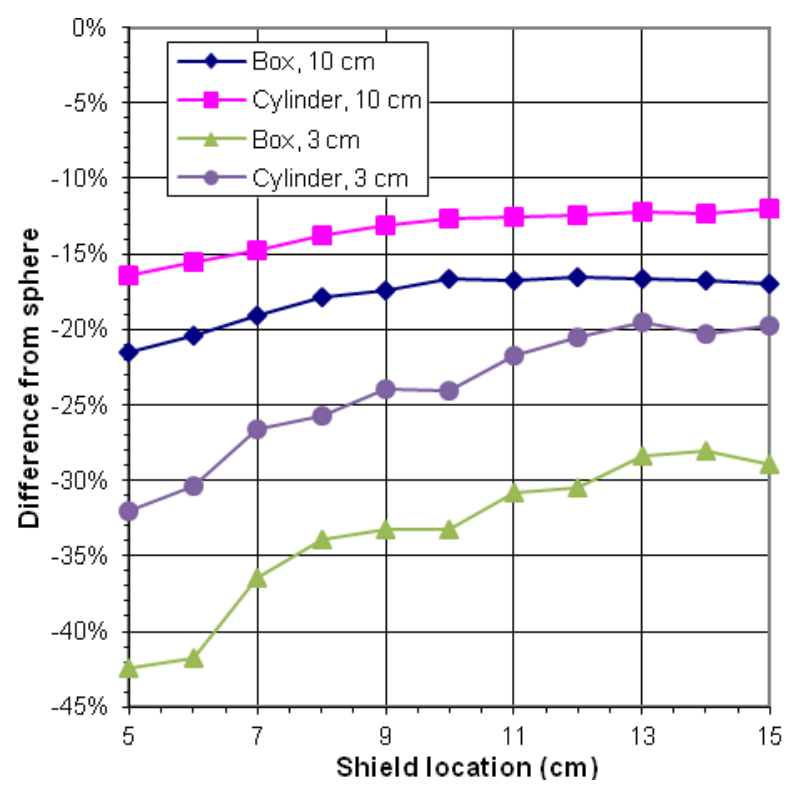

Figure 6. Difference between 1.8848-MeV line fluxes from multidimensional and spherical C-H-N-O shields.

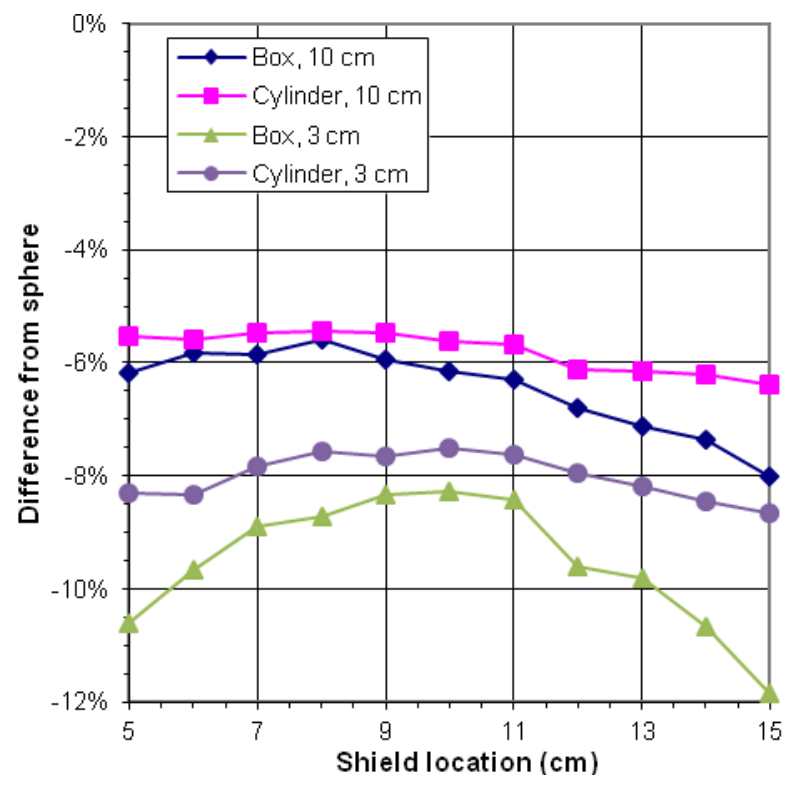

Figure 7. Difference between 4.4390-MeV line fluxes from multidimensional and spherical C-H-N-O shields. 
thickness $t$ that minimized the $\chi^{2}$ difference [Eq. (1)] between the Monte Carlo (reference) leakages and leakages from the spherical object, which were calculated using the discrete-ordinates code PARTISN [6] (Version 6.52) with $S_{32}$ quadrature, $P_{3}$ scattering, and the 250-group ACTI library [5], which has 130 neutron groups (52 of which include upscattering) and 120 photon groups with tight energy bins around the lines of interest. The four lines of Table 1 were used in the optimization, so $G=4$ in Eq. (1).

The difference between the optimal spherical shield thickness (in terms of matching the neutron-induced line fluxes) and the assumed sphere of thickness $t$ (either 3 or $10 \mathrm{~cm}$ ) is plotted as a function of shield location $d$ in Figure 8 for the two multidimensional geometries. The difference was as large as $50 \%$. The difference increases with shield location when the shield is thick $(10 \mathrm{~cm})$ but decreases with shield location when the shield is thinner $(3 \mathrm{~cm})$.

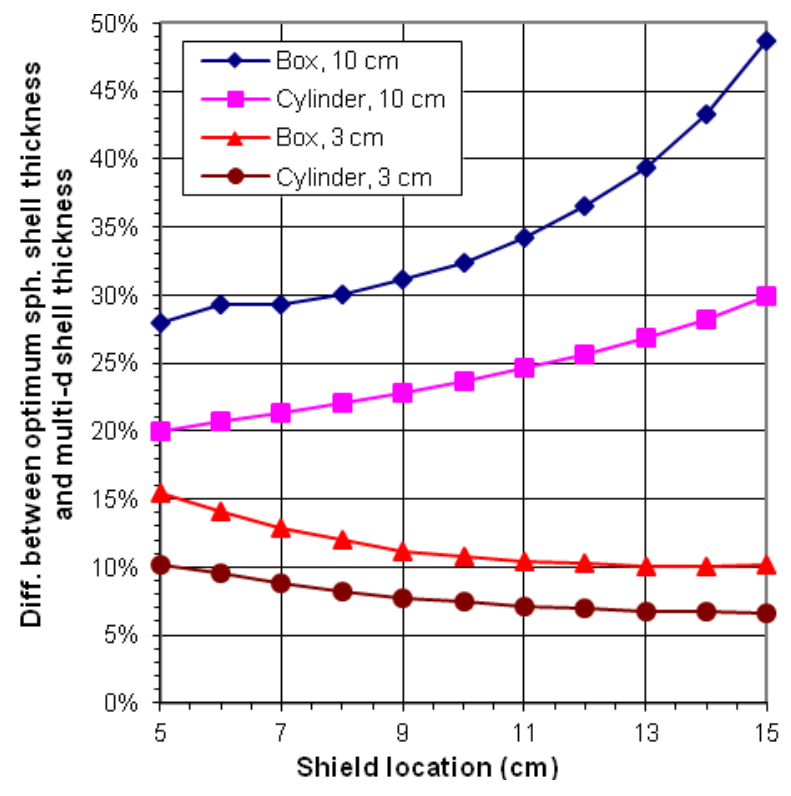

Figure 8. Difference between the optimum spherical shield thickness and the multidimensional shield thickness, relative to the latter, using four neutron-induced gamma-ray lines from C-H-N-O.

\section{Conclusion}

The simple substitution of a spherical shield for a flat or cylindrical shield in order to do one-dimensional calculations for detected passive or neutron-induced gamma-ray line fluxes may lead to significant errors in the computed fluxes. "Simple" refers to the practice of maintaining the location and thickness of the multidimensional shield, but making it a sphere.

For uncollided passive gamma rays, this type of spherical approximation is most appropriate for optically thin shields far from the source; it may be quite inappropriate for optically thick shields or any shield close to the source.

For neutron-induced gamma rays, this type of spherical approximation is more appropriate for thick shields than for thin shields, but it may still be quite inappropriate; it is worse for thermal capture lines than inelastic scattering lines. For both types of gamma rays, the spherical approximation is worse for flat shields than cylindrical.

It is often noted that the farther away a detector is, the more a source/shield system looks like a point. In the figures in this paper, the detector location is fixed. It is found that the farther away a box or cylindrical shield is from the source, the more the entire system looks like a one-dimensional sphere. Conversely, when a box or cylindrical shield is close to the source, large errors will be made if the shield is modeled as a sphere, even if the source itself is spherical.

Complete results of these calculations are available $[2,7]$.

\section{References}

[1] X-5 Monte Carlo Team, MCNP - A General N-Particle Transport Code, Version 5 - Volume I: Overview and Theory, LA-UR-03-1987, Los Alamos National Laboratory (2003).

[2] J.A. Favorite, Comparing Uncollided Gamma-Ray Fluxes through Multidimensional and Equivalent One-Dimensional Shields, LA-UR-11-5044, Los Alamos National Laboratory (Aug. 29, 2011).

[3] J.A. Favorite, K.C. Bledsoe and D.I. Ketcheson, Surface and volume integrals of uncollided adjoint fluxes and forward-adjoint flux products, $\mathrm{Nucl}$. Sci. Eng. 163 (2009), pp. 73-84.

[4] K.C. Bledsoe, J.A. Favorite and T. Aldemir, Using the Levenberg-Marquardt method for solutions of inverse transport problems in one- and two-dimensional geometries, Nucl. Technol. 176 (2011), pp. 106-126.

[5] S. Frankle, A prototype fine-group library for gamma-ray spectroscopy, ACTI, Internal Memorandum XCI:SCF-99-19(U), Los Alamos National Laboratory (updated Feb. 2, 2004).

[6] R.E. Alcouffe, R.S. Baker, J.A. Dahl, S.A. Turner and R.C. Ward, PARTISN: A time-dependent, parallel neutral particle transport code system, LA-UR-08-7258, Los Alamos National Laboratory (Nov. 2008).

[7] J.A. Favorite, Comparing Neutron-Induced Gamma-Ray Fluxes through Multidimensional and Equivalent One-Dimensional Shields, LA-UR-11-7041, Los Alamos National Laboratory (Dec. 14, 2011). 